\title{
RETRACTION
}

\section{Impact of Renin-Angiotensin System Inhibition on Microalbuminuria in Type 2 Diabetes: A Post Hoc Analysis of the Shiga Microalbuminuria Reduction Trial (SMART)}

The Shiga Microalbuminuria Reduction Trial (SMART) Group

Hypertension Research (2014) 37, 598; doi:10.1038/hr.2014.69; published online 27 March 2014

Retraction to: Hypertension Research (2008) 31, 1171-1176; doi:10.1291/hypres.31.1171

The authors have indicated to the journal that this paper should be withdrawn in response to the interim report on the Shiga Microalbuminuria Reduction Trial (SMART), and in consequence of the retraction of the main report from the same trial. ${ }^{1}$
After careful consideration, Hypertension Research editorial committee formally retracts this paper with agreement of the authors.

1 The Shiga Microalbuminuria Reduction Trial (SMART) Group. Reduction of microalbuminuria in patients with type 2 diabetes: The Shiga Microalbuminuria Reduction Trial (SMART). Diabetes Care 2007; 30: 1581-1583. 